\title{
Narrativa
}

\section{Riccardo CASTELLANA, Finzioni biografiche. Teoria e storia di un genere ibrido}

Irene Cecchini

\section{OpenEdition}

\section{Journals}

Edizione digitale

URL: https://journals.openedition.org/narrativa/304

DOI: $10.4000 /$ narrativa.304

ISSN: 2804-1224

\section{Editore}

Presses universitaires de Paris Nanterre

\section{Edizione cartacea}

Data di pubblicazione: 1 décembre 2020

Paginazione: 194-195

ISBN: 978-2-84016-381-7

ISSN: $1166-3243$

\section{Notizia bibliografica digitale}

Irene Cecchini, «Riccardo castellana, Finzioni biografiche. Teoria e storia di un genere ibrido», Narrativa [Online], 42 | 2020, online dal 01 novembre 2021, consultato il 08 décembre 2021. URL: http:// journals.openedition.org/narrativa/304 ; DOI: https://doi.org/10.4000/narrativa.304

Questo documento è stato generato automaticamente il 8 décembre 2021

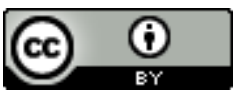

Narrativa est mise à disposition selon les termes de la Licence Creative Commons Attribution 4.0 International. 


\title{
Riccardo CASTELLANA, Finzioni biografiche. Teoria e storia di un genere ibrido
}

\author{
Irene Cecchini
}

\section{NOTIZIA}

Riccardo CASTELlana, Finzioni biografiche. Teoria e storia di un genere ibrido, Roma, Carocci, 2019, pp. 216.

1 Con Finzioni biografiche, Castellana vuole riempire un vuoto critico, collocandosi nello spazio indistinto fra il "tutto è finzione" postmoderno e il presupposto "ritorno al Reale" contemporaneo. Castellana si inserisce con intelligenza all'interno del dibattito narratologico tra la natura della fiction e della non fiction con uno studio millimetrico sulla biofinzione: una "finzione narrativa in prosa incentrata sulla vita di una persona reale, distinta dall'autore" (p. 36), la cui divergenza con la biografia ha origine dalla combinazione "di tangenze e sovrapposizioni tra fiction e il mondo reale" (p. 12).

2 Riesaminando la linea Hamburger-Genette-Cohn, Castellana non si ferma alla centralità dell'aspetto puramente modale della narrazione, ma approfondisce lo studio della biofinzione attraverso il paratesto e il patto di lettura. Se in una prima fase l'autore rischia di privilegiare un'attitudine classificatoria, in seguito riconosce il "paradosso" di questo genere ibrido e si cala nel "terreno della storia" (p. 90) per osservare le scelte poetiche di singoli scrittori.

3 In una prima parte, Problemi e modelli, Castellana descrive l'ibridismo di queste "storie vere", analizzando il modo stesso di scrivere la finzione: l'autore identifica così le strategie discorsive che reinventano la vita reale. Il modello teorico segue precisi criteri narratologici, come il punto di vista e la posizione del narratore, e arriva a costituire una serie di tipologie possibili in cui suddividere il macro-concetto di biofiction. Il campione testuale osservato dall'autore è convincente e frutto di numerose letture, e la 
classificazione minuziosa - Castellana suddivide la biofiction in eterobiofiction, omobiofiction, metabiofiction - testimonia una forte attenzione verso le strategie formali e verso elementi pragmatici, anche se talvolta rischia di disorientare il lettore (la metabiografia in 5 varianti diverse ne è un esempio).

4 La storia del genere è ripercorsa nella seconda parte, Temi e tradizioni, ripartita cronologicamente; qui Castellana ne racconta l'origine ed elegge alcuni modelli biofinzionali (dal protogenere Vite immaginarie di Schwob, fino alle tre corone francesi Michon, Echenoz e Carrère), per poi soffermarsi sul panorama italiano (Del Giudice, Tabucchi, Orecchio tra altri). Così facendo Castellana conferma la non esclusiva appartenenza del genere biofinzionale al postmoderno, e sviluppa un'interessante riflessione sulla distinzione stilistica e culturale fra la biofiction postmoderna e ipermoderna attraverso un'analisi approfondita di Mari e di Balestrini.

Lo studio di Castellana si chiude su alcuni interrogativi riguardo alla funzione conoscitiva ed etica della letteratura - sulla scia di alcune riflessioni di Donnarumma e sulla capacità della biofiction di cogliere il senso di un'epoca attraverso la lente della coscienza individuale.

6 Il saggio riesce nella mappatura di un genere "dai confini mobili ma tutt'altro che indefiniti e illusori" (p.192) e ammonisce la critica letteraria a bilanciare uno studio sistematico del corpus con una apertura alle singole varianti poetiche. Se in Italia il genere biofinzionale restava ancora inesplorato, Finzioni biografiche ne riconosce "la forma simbolica di lunga durata" (p. 192). 